\section{$(3)$ \\ HORIZONTES}

www.revistahorizontes.org
Horizontes. Revista de Investigación en Ciencias de la Educación Https://doi.org/10.33996/revistahorizontes.v4i13.88

Enero - Marzo 2020

Volumen 4 / No. 13

ISSN: 2616 - 7964

pp. 15 - 29

\title{
Estrategias y actividades para aprendizaje del léxico en dos manuales escolares de educación primaria
}

\author{
Strategies and activities for lexicon learning in two primary school \\ handbooks
}

\section{Estratégias e atividades para a aprendizagem de léxico em dois manuais da escola primária}

\author{
Ángel Delgado Jiménez 1-2 \\ adelgado95@gmail.com
}

Endri González Romero ${ }^{1}$

alfonsogr1976@hotmail.com

\author{
Donaldo García Ferrer ${ }^{1}$ \\ donaldogf@gmail.com
}

\author{
Gisela Swiggers ${ }^{1}$ \\ giselaswiggers@hotmail.com
}

Universidad del Zulia, Venezuela ${ }^{1}$

Universidad Católica Cecilio Acosta, Venezuela²

Recibido octubre 2019 | Revisado nov - dic 2019 | Publicado 01 de enero 2020

\section{RESUMEN}

El trabajo tuvo como objetivo analizar las estrategias y las actividades para el aprendizaje del léxico, utilizadas por dos manuales escolares de la escuela primaria venezolana. Se sustentó en Rodríguez (2006), Giammatteo y otros (2009) y Moreno (2004). La investigación fue de tipo descriptivo-comparativa con un diseño documental. El corpus estuvo conformado por 306 actividades extraídas de doce textos, de primero a sexto grado, del área de lengua y literatura. La técnica utilizada fue el análisis de contenido y como instrumento, una base de datos. El procedimiento estadístico fue la prueba de frecuencia. Se concluye que en los textos prevalecen estrategias $\mathrm{y}$ actividades memorísticas, descontextualizadas y convencionales; enmarcadas en el enfoque tradicionalista de le lengua, en el que la enseñanza del vocabulario es considerada de forma marginal. Por lo tanto se requiere de un cambio significativo en la gestión didáctica que favorezca el desarrollo de la competencia léxica.

Palabras clave: Enseñanza de la lengua; competencia comunicativa, competencia lingüística; gestión pedagógica; gestión del aprendizaje; léxico

\section{ABSTRACT}

The objective of this study was to analyze the strategies and activities for learning vocabulary, used by two school manuals of the Venezuelan primary school. It was based on Rodríguez (2006), Giammatteo and others (2009) and Moreno (2004). The research was descriptive-comparative with a documentary design. The corpus was made up of 306 activities from twelve texts, from first to sixth grade, in the area of language and literature. The technique used was content analysis and as an instrument, a database. The statistical procedure was the frequency test. It is concluded that the texts prevail strategies and memoristic, decontextualized and conventional activities; framed in the traditionalist approach of the language, in which the teaching of vocabulary is considered marginally. Therefore, a significant change in didactic management is required that favors the development of lexical competence.

Key words: Language teaching; communicative competence; linguistic competence; pedagogical management; learning management; lexicon 


\section{RESUMO}

0 trabalho teve como objetivo analisar as estratégias e atividades de aprendizagem de vocabulário utilizadas por dois manuais escolares da escola primária venezuelana. Foi baseado em Rodríguez (2006), Giammatteo e outros (2009) e Moreno (2004). A pesquisa foi descritivacomparativa com delineamento documental. 0 corpus foi composto por 306 atividades, extraídas de doze textos, da primeira à sexta série, na área de língua e literatura. A técnica utilizada foi a análise de conteúdo e, como instrumento, um banco de dados. 0 procedimento estatístico foi o teste de frequência. Conclui-se que os textos predominam estratégias e atividades memorísticas, descontextualizadas e convencionais; enquadrado na abordagem tradicionalista da língua, na qual o ensino do vocabulário é considerado marginalmente. Portanto, é necessária uma mudança significativa no gerenciamento didático que favorece 0 desenvolvimento da competência lexical.

Palavras-chave: Ensino de línguas; competência comunicativa; competência linguística, gestão pedagógica; gestão da aprendizagem; léxico

\section{INTRODUCCIÓN}

El niño inicia la adquisición $y$ conformación de su vocabulario a partir del uso de palabras aisladas que luego pondrá en contexto y le permitirán conformar su competencia léxica que se concibe como un subcomponente de la competencia comunicativa. A juicio de Cassany, Luna y Sanz (2001), la enseñanza de la lengua debe considerar el aprendizaje del léxico, en forma paralela a la adquisición del contacto con su entorno y con el resto de las áreas aprendizaje. Por lo tanto su función no es ampliar el componente vocabular del estudiante sino también su conocimiento sobres las características y funciones de las palabras como unidades léxicas.

Todo esto hace necesario la reorientación en la gestión didáctica, en especial en la determinación de las estrategias y las actividades. Se podría decir que el enfoque es teórico y los métodos son procedimentales. de la enseñanza del léxico en la escuela primaria, puesto que en Venezuela, se ha asumido la premisa de que el niño va conformando su parcela vocabular $y$, por ende, su enriquecimiento, mediante la interacción verbal que haga en su entorno. Esta concepción ha relegado la enseñanza de léxico a las aulas de segundas lenguas (L2), bien sea como L2 propiamente dicho o como lenguas extranjeras (LE), ya que el hablante focaliza en el léxico sus necesidades de comunicación. Las observaciones empíricas y la experiencia en aula de los investigadores les han permitido detectar que en la enseñanza del léxico en el aula venezolana se recurre, exclusivamente, a actividades convencionales, en algunos casos lúdicas que se caracterizan por ser memorísticas y descontextualizadas. La presente investigación tiene como objetivo analizar las estrategias y las actividades para el aprendizaje del léxico, utilizadas por dos manuales escolares de la escuela primaria venezolana.

\section{Fundamentación teórica}

\section{Enseñanza del léxico}

En el proceso de enseñanza y aprendizaje, existen un conjunto de principios metodológicos que les permiten a los docentes sistematizar dicho procesos, entre los que se encuentra los términos enfoques y métodos. Ambos tienden a confundirse, Según Cortés (2000), el enfoque plantea los principios epistemológicos y pedagógicos sobre la enseñanza y el aprendizaje del objeto de estudio, en este caso la lengua $y$, específicamente, el léxico; mientras que el método alude a los principios metodológicos que rigen la actividad en el aula. Estos inciden

En el caso del léxico, su enseñanza ha estado vinculada a la enseñanza de la lengua en general. El tratamiento que se le ha dado ha dependido si el aula es de lengua materna 
(LM) o segundas lenguas (L2). En el caso de LM el interés es menor ya que los diversos enfoques y métodos ha privilegiado o bien la gramática o bien las cuatro macrohabilidades de la lengua (leer, hablar, escuchar o escribir). En lo que respecta a las aulas de L2 o LE, el interés ha sido distinto ya que, como plantea Vidiella (2012) el aprendizaje del léxico le permite al estudiante, en principio, aprender a comunicarse y, luego, cuando incrementa su vocabulario, le permitirá tener una mayor conciencia del léxico y de la gramática de la lengua que aprende.

El tratamiento del léxico en los principales enfoque utilizado en la escuela es diverso. En el método tradicional o gramaticalista que no tiene sustento en ninguna teoría lingüística ni psicológica, se enseña el vocabulario escrito mediante listas y glosarios con actividades descontextualizadas y un aprendizaje memorístico. Por su parte, el método estructuralista, sustentado en la lingüística estructuralista americana o europea, concibe la lengua como un sistema de elementos relacionados y el léxico deriva de la construcción sintáctica. La teoría psicológica conductista que lo soporta considera que el aprendizaje de la lengua se basa en la producción de respuestas a estímulos lingüísticos. Al igual que el gramaticalista, se enseña el vocabulario escrito mediante listas y glosarios con actividades descontextualizadas y aprendizaje memorístico.

El método comunicativo-funcional que se fundamenta en la lingüística funcional, la lingüística textual y la pragmática, prioriza la lengua con fines comunicativos y promulga el desarrollo de la competencia comunicativa. En este método, el aprendizaje de la lengua es una construcción del aprendiz en función de sus conocimientos previos y se enseña el vocabulario de acuerdo con las exigencias del acto comunicativo. Se valora la planificación léxica y su selección obedece en criterios temáticos. Finalmente, se encuentra el enfoque basado en estrategias múltiples, propuesto por Giammateo y otros (2009) y se sustenta en el enfoque comunicativo funcional. Su finalidad es el desarrollo del léxico y el incentivo de la lectura y la escritura de textos disciplinares y literarios; así como promover la capacidad integradora del significado de las palabras disciplinares y operativas que se involucran en los procesos de comprensión y producción de textos y el desarrollo de las destrezas representacionales que se establece por medio del léxico y el contexto.

Los tres primeros enfoques consideran el léxico en forma marginal, bien subordinada a la sintaxis o al desarrollo de la competencia comunicativa, solo el enfoque propuesto por Giammateo y otros (2009) le otorga relevancia para el desarrollo de la lectura y la escritura, ya que "no es posible realizar una propuesta relativa al vocabulario separada de su contexto de uso que solo podemos aislarlo con fines operativos, para luego reintegrarlo al texto en el que la palabra encuentra su significación y su utilidad" (p. 33).

Además de los enfoques, en la enseñanza del léxico también se presentan dos formas de aprendizajes: el explícito o directo y el implícito o indirecto. El primero propone una enseñanza intencionada del léxico, mediante el estudio de contenidos y desarrollo de actividades relacionadas con el vocabulario en sus diferentes niveles: ortográfico, fonológico y morfológico, sintáctico o semántico. El aprendizaje implícito o intencional, es quizás el más tradicional y se logra mediante el desarrollo de contenidos o actividades que requiera del estudiante conocer el significado de ciertas lexías desconocidas, bien sea mediante el contexto o por el diccionario. Para Nation (2001), "incidental learning guessing from context is the most important of all sources of vocabulary learning. This is particulary true for native speakers learning their first 
language ${ }^{1 "}$. En este tipo de aprendizaje el docente se vuelve un modelo para el desarrollo del léxico.

Como plantea Rodríguez (2006) ambas tendencias no son excluyentes. Por el contrario se complementan y permiten compensar deficiencias que conllevan de un estudio descontextualizado y aislado:

En numerosas ocasiones, resulta inevitable desarrollar explícitamente ciertos aspectos del vocabulario, en particular, los relacionados con el contenido y con la derivación: los contextos en los que se insertan las piezas léxicas no siempre permiten la inferencia de este tipo de información (semántica y gramatical). Se hace necesaria, en tal caso, una reiterada, y no siempre viable, exposición al mismo vocabulario, en diferentes marcos discursivos. (p. 105).

En El Currículo Nacional Bolivariano (CNB, 2007b) no establece en forma explícita el enfoque para la enseñanza de la lengua. El CNB y, por ende, al Currículo para el Subsistema de Educación Primaria (CSEP, 2007a) se asocia al Enfoque Comunicativo Funcional, no de forma patente, ya que los redactores se cuidan en establecer etiquetas, pero en algunos párrafos de los distintos componentes del currículo, en especial, cuando se define el área de aprendizaje de Lengua, Comunicación y Cultura se desdibuja la relación que hay con los principios generales del enfoque:

4.1- Lenguaje, Comunicación y Cultura Con esta área se aspira que niños y niñas desarrollen potencialidades que les permitan, como seres sociales y culturales, promover variadas $\mathrm{y}$

\footnotetext{
${ }^{1}$ El aprendizaje incidental del léxico es supuestamente la fuente más importante para el aprendizaje del vocabulario. Esta premisa resulta válida para el aprendizaje de hablantes nativos en su lengua materna (Traduc. del autor).
}

auténticas experiencias comunicativas, participativas donde expresen y comprendan mensajes, logrando una comunicación efectiva al expresar sus necesidades, intereses, sentimientos y experiencias en la familia, escuela y comunidad, respetando la diversidad en los códigos lingüísticos, fortaleciendo hábitos efectivos de lectura $y$ afianzando el proceso productivo de la lengua (hablar y escribir), con énfasis en idioma materno (castellano e indígena) y los receptivos (escuchar y leer), partiendo del hecho que el lenguaje está predeterminado por el contexto histórico social y cultural, como vía para ampliar el horizonte cultural e intelectual con el conocimiento del otro. (Ministerio del Poder Popular para la Educación; 2007a p. 21)

Esto coincide con lo planteado por el CSEP, en el perfil de egreso en donde se plantea, entre otros aspectos, que el egresado de educación primaria debe tener «habilidades de lectura y escritura, interpretativa y crítica» (Ministerio del Poder Popular para la Educación; 2007a). Además en las orientaciones pedagógicas del Currículo Nacional Bolivariano (2007b) también plantea que en lo que respecta a las experiencias de aprendizajes:

Se concibe que éstas deben propiciar una relación comunicativa y potenciadora de la capacidad de resolver problemas, a través de la cual los actores del hecho educativo experimenten constantemente para movilizar sus procesos y operaciones mentales, partiendo de la confrontación teórica de la realidad y, sobre todo, nutriéndose de sentimientos de amor, humildad y confianza en el diálogo; para que así se fortalezca una toma de conciencia basada en lo que se aprende. (Ministerio del Poder Popular para la Educación; 2007b:50) 
En lo que respecta al léxico se introduce a partir de las necesidades y requerimientos comunicativos. El CSEP presenta una serie de núcleos temáticos que están relacionados con el léxico en cualquiera de sus niveles. Dichos contenidos se presentan en el cuadro 1.

Cuadro 1. Contenidos relacionados con el léxicos en el CSEP

\section{Contenidos}

Decodificación de símbolos para darles significados como palabras

\section{Grado según currículo}

1 ero

Enriquecimiento de vocabulario / Enriquecimiento del vocabulario a partir de la $1^{\text {ero }}, 2^{\text {do }}, 3^{\text {ero }}$

de prácticas para el enriquecimiento del vocabulario /

lectura de textos cortos.

Construcción de familias de palabras / Elaboración de familias de palabras para la construcción de oraciones

$1^{\text {ero }}, 2^{\text {do }}, 3^{\text {ero }}$

Reproducción y juegos de palabras

$2^{\text {do }}$

Establecimiento o identificación del significado social de las palabras.

$2^{\text {do }}, 3^{\text {ero }}$

Construcción de significados sobre el comportamiento de las personas

$2^{\text {do }}$

Problematización de los significados de las palabras, frases y oraciones

$2^{\text {do }}, 3^{\text {ero }}$

Diferenciación de sinónimos y antónimos / Aplicación de sinónimos y antónimos para construir pequeños textos/ Aplicación de sinónimos, antónimos y $2^{\text {do }}, 3^{\text {ero }}, 4^{\text {to }}$ homónimos para la escritura de párrafos cortos

Utilización de sustantivos y adjetivos para el enriquecimiento del vocabulario

$2^{\text {do }}$

Identificación de prefijos y sufijos / Utilización de prefijos y sufijos para construir nuevas palabras.

$2^{\text {do },} 3^{\text {ero }}$

Utilización del libro y del diccionario. Identificación de Tipos y partes del libro / Uso del libro y el diccionario como herramienta de investigación / Apropiación al manejo del diccionario

Fuentes: Currículo para el Subsistema de Educación Primaria (2007a)

\section{Estrategias para el aprendizaje del léxico}

Las estrategias son otro componente importante de la gestión didáctica y están vinculadas con el enfoque y el método de enseñanza y aprendizaje general y especifico de cada disciplina. El currículo debe brindarle al docente un espectro de los tipos de estrategias que puedan ser utilizadas para el desarrollo del proceso educativo. Según DíazBarriga y Hernández (2002) se pueden concebir como "«procedimientos flexibles, heurísticos (nunca como algoritmos rígidos) y adaptables, dependiendo de los distintos dominios del conocimiento, contextos o demandas de los episodios o secuencias de enseñanza de que se trate". 
Esta concepción de estrategias considera al docente $\mathrm{y}$ al estudiante como entes reflexivos y estratégicos, capaces de seleccionar las estrategias en función del contexto de enseñanza y aprendizaje con el fin de promover aprendizajes significativos de calidad. Díaz-Barriga y Hernández (2002) distinguen entre estrategias de enseñanza y de aprendizaje. Las primeras, siguiendo a Meyer, Shuell, West, Framer y Wolf, las define como los procedimientos que permiten promover el logro de aprendizajes significativos en los alumnos; y las segundas son las que utilizan los aprendices para aprender, recordar o solucionar algún problema relacionado con los contenidos de los aprendizajes. Para Brown y para Flavell y Wellman citados por Díaz-Barriga y Hernández (2002), los tipos de conocimientos a los que se enfrentan los aprendices son: a) procesos cognitivos básicos, b) conocimientos conceptuales específicos; c) conocimientos $\begin{array}{lll}\text { estratégicos } & \mathrm{y} & \mathrm{d}) \\ \end{array}$ metacognitivos.

Los tipos de estrategias de enseñanzas y de aprendizajes son muy variadas. Las de enseñanzas las clasifica en tres: 1) generales de enseñanza, 2) según el episodio del proceso de enseñanza y aprendizaje y 3) según el proceso cognitivo atendido. Las de aprendizaje, también las ordena en tres grupos: 1) las generales de aprendizajes, 2) las de aprendizajes para contenidos declarativos de tipo factual y 3) las referidas a contenidos declarativos complejos. En este trabajo se consideraron como indicadores de análisis las estrategias de aprendizajes referidas a los conocimientos conceptuales específicos que propone Rodríguez (2006).

Según el autor, para el aprendizaje del léxico se recurren a tres tipos de estrategias: a) las cognitivas, b) las comunicativas o de compensación y c) las memorísticas. La primera "se destinan a la manipulación consciente de la lengua para propiciar el aprendizaje" y las comunicativas "suponen la aplicación de técnicas que permiten compensar los problemas que dificultan los intercambios comunicativos" (Rodríguez, 2006). Por su lado, las memorísticas tienen como propósito:
"la recuperación del léxico, la recepción o comprensión y el almacenamiento mental de las palabras. Tienen por objetivo facilitar la comprensión de los términos desconocidos que surgen en el discurso y, al mismo tiempo, propiciar el reconocimiento de nuevos usos y significados" (p 134).

\section{Actividades para el aprendizaje del léxico}

Para Cortés (2002), las actividades son uno de los componentes esenciales de las tareas que son definidas como "la labor de comprensión y/o producción en la LM que realizan unos aprendientes siguiendo unas instrucciones, y que aspiran, en última instancia, al desarrollo de unos aspectos concretos [...] ". Son las más variadas y dependen, en parte, de la creatividad del docente. Para la enseñanza del léxico se han desarrollado un conjunto de actividades. Casanny y otros (2001) no han establecido una tipología respectiva sino que han inventariado un conjunto de actividades, entre las que se encuentran: a) escribir la palabra, b) escribir su traducción, c) escribir su definición, d) relacionarla con otras palabras, e) intentar recordar cómo suena, f) asociar su sonido al de otras palabras, g) repetirlas mentalmente, $h$ ) pensar en un contexto en donde pueda ser utilizada, i) asociarla con un sinónimo o antónimo, j) formarse una imagen mental de la palabra o del objeto, k) hacer un dibujo o gráfico. 
Las actividades propuestas por los autores se caracterizan por ser memorísticas, descontextualizadas y permiten poco desarrollo de la competencia léxica. Moreno (2004), tomando como sustento teórico el enfoque léxico de Lewis, que según Peñalver (1991) tiene como objetivo "reconocer la importancia de la semántica y ponerla como punto de partida en lugar de punto de llegada"; organiza las actividades que tradicionalmente han sido usadas para la enseñanza del léxico en cuatro grupos las convencionales, las lúdicas, las de dramatización y las de constelación. La primera las define como el conjunto de ejercicios de vocabulario que tienden a ser de resolución individual, poco contextualizados y algo mecánicos. Son los más usados por la tradición escolar. Entre las actividades se destacan la clasificación del vocabulario según la clase de palabras o partes de la oración, la relaciona las palabras con su definición, la organización de vocablos por orden alfabético, las familias léxicas, los campos semánticos, sinónimos o antónimos, la construcción de frase a partir de vocablos y la búsqueda en diccionarios.

Las actividades lúdicas son ejercicios que recurren al juego y al componente cooperativo para su realización. Presentan poca contextualización, entre las actividades más usuales se encuentran las sopas de letras, los crucigramas, las palabras cruzadas (imagen-vocablo), cruzaletras, formar palabras con las letras de una palabra, la palabra oculta, construcción de un acróstico y los pictograma (plasma con un dibujo una de las palabras).

Las actividades de constelación y las de dramatización permite dar cuenta de las múltiples relaciones que se establecen entre los vocablos, además de garantizar "la realización de tareas y ejercicios de escritura y de expresión oral que garanticen la memorización del nuevo vocabulario, al tiempo que practican las cuatro destrezas básicas de la lengua: producción y recepción oral y escrita" (Moreno; 2004). Las de constelación tienen como propósito producir palabras, a partir de un vocablo generador (input). Los vocablos van emergiendo a partir de relaciones lingüísticas de tipo fonetológicas, morfológicas y léxicosemánticas. La tarea se puede resolver en pequeños grupos de cuatro, o bien de manera conjunta por el grupo-aula en la pizarra, con el correspondiente apoyo docente. Con este vocabulario surgido se pasa a tareas de escritura, de expresión oral. Las actividades se sustentan en realizar, en forma oral o escrita, a partir de un vocabulario aislado, una tarjeta postal, una noticia, un anuncio para un cartel publicitario, un minirelato según la técnica del binomio fantástico, una miniconferencia (uno o dos minutos), contar un recuerdo personal, un viaje, una anécdota, la entrevista a un personaje famoso, o un telediario con noticias escolares.

Las actividades de dramatización integran el vocabulario tanto en el lenguaje verbal como en el no verbal, por medio de la escenificación de situaciones comunicativas específicas. Con ellas se ponen en práctica las destrezas comunicativas para solventar distintas situaciones. Ejercitan simultáneamente el plano intelectual, el imaginativo, el motor y el afectivo, dado que interactúan en una situación virtual. Tienen un carácter lúdico y entre ellas se encuentran los juegos de puesta en marcha, los de vocalizaciones con algunas de las palabras, los de expresión corporal y las escenificaciones de situación conflictiva sugerida por el vocabulario. 
MÉTODO

La presente investigación es de tipo descriptiva-comparativa, con un diseño documental. El corpus está conformado 306 actividades, extraídas de doce textos, de primero a sexto grado, del área de lengua y literatura de la serie El Cardenalito, Lengua y Literatura de la Colección Bicentenario y la Enciclopedia Girasol. Los datos relacionados con los textos analizados se muestran en el cuadro 2. La técnica de recolección de datos fue el análisis de contenido y como instrumento se diseñó una base de datos cuyos campos fueron: 1) tipo de texto, 2) año de edición, 3) total de página y/o total de página de la sección, 4) total de actividades y/o total de actividades por sección, 5) tipo de enseñanza del léxico (implícito y explícito), 6) tipo de actividades y 7) tipo de estrategias de para el aprendizaje del léxico. $\mathrm{El}$ procesamiento estadístico que se utiliza fue la estadística descriptiva, prueba de frecuencia.

Cuadro 2. Datos de los textos analizados

\begin{tabular}{|c|c|c|c|c|c|}
\hline Texto & Editorial & Grado & Año de edición & $\begin{array}{l}\text { Total de } \\
\text { págs. }\end{array}$ & $\begin{array}{c}\text { Págs. de la } \\
\text { sección }\end{array}$ \\
\hline El Cardenalito 1 & & $1^{\text {ero }}$ & 2013 & 70 & 0 \\
\hline El Cardenalito 2 & & $2^{\text {do }}$ & 2014 & 159 & 0 \\
\hline El Cardenalito 3 & $\begin{array}{l}\text { Ministerio del } \\
\text { Poder Popular para }\end{array}$ & $3^{\text {ero }}$ & 2012 & 159 & 0 \\
\hline El Cardenalito 4 & la Educación & $4^{\text {to }}$ & 2013 & 175 & 0 \\
\hline El Cardenalito 5 & & $5^{\text {to }}$ & 2013 & 176 & 0 \\
\hline El Cardenalito 6 & & $6^{\text {to }}$ & 2012 & 191 & 0 \\
\hline Enciclopedia Girasol 1 & & $1^{\text {ero }}$ & 2008 & 331 & 65 \\
\hline Enciclopedia Girasol 2 & & $2^{\text {do }}$ & 2007 & 360 & 51 \\
\hline Enciclopedia Girasol 3 & Editorial Girasol & $3^{\text {ero }}$ & 2013 & 383 & 57 \\
\hline Enciclopedia Girasol 4 & & $4^{\text {to }}$ & 2013 & 423 & 75 \\
\hline Enciclopedia Girasol 5 & & $5^{\text {to }}$ & 2014 & 431 & 87 \\
\hline Enciclopedia Girasol 6 & & $6^{\text {to }}$ & 2014 & 520 & 97 \\
\hline
\end{tabular}

\section{RESULTADOS}

El primer indicador analizado fueron las estrategias de aprendizajes del léxico propuestas por Rodríguez (2006). Los resultados nos permiten afirmar que 268 actividades relacionadas con el léxico son de tipo memorísticas, al discriminarse por colección, 166 (82.18\%) se encuentran en la
Enciclopedia Girasol y 102 (98.08\%) en la Colección Bicentenario. Le siguen las comunicativas o de compensación, repartidas de la siguiente manera: 36 (17.82\%) en Girasol y 2 (1.92\%) en El Cardenalito. Las estrategias cognitivas no aparecieron en ninguna de las actividades (Ver gráfico 1). 


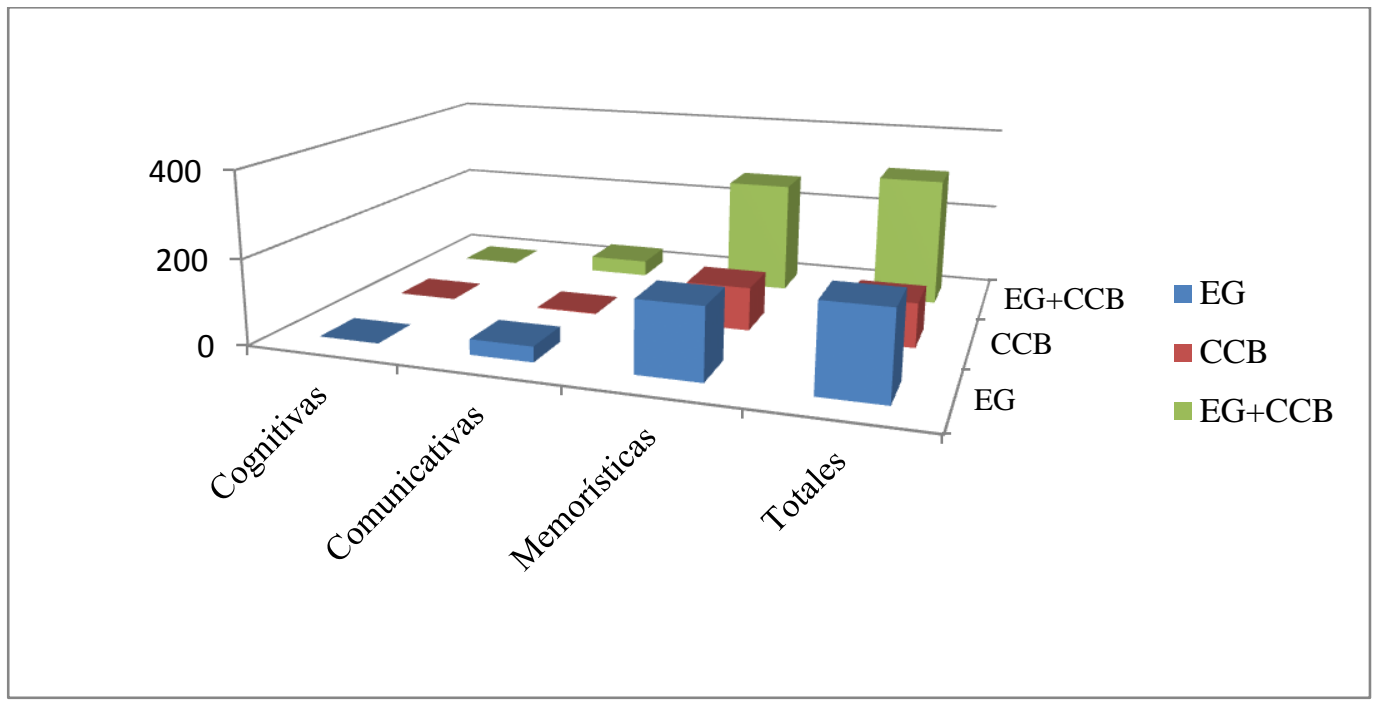

Gráfico 1. Tipo de estrategias de aprendizaje del léxico

Los resultados permiten afirmar que las actividades para la enseñanza del léxico presente en los textos escolares venezolanos tienen como propósito la memorización de listas de palabras o el desarrollo de actividades descontextualizadas. Esto trae como consecuencia una enseñanza tradicional y un aprendizaje fragmentado sin conexiones con la lectura ni la escritura, lo que no permite el enriquecimiento del vocabulario ni el desarrollo de la competencia léxica. Esta situación es contradictoria con lo expresado en el currículo para Subsistema de Educación Primaria (CSEP, 2007a) tenga un enfoque comunicativo-funcional que busca "promover variadas y auténticas experiencias comunicativas, participativas donde expresen y comprendan mensajes, logrando una comunicación efectiva al expresar sus necesidades, intereses, sentimientos y experiencias en la familia, escuela $y$ comunidad, respetando la diversidad en los códigos lingüísticos" (Ministerio del Poder Popular para la Educación; 2007a).
Para analizar las actividades que están presentes en los textos, primero se procedió a determinar al número de actividades relacionadas con el léxico que se encuentran en ellos. La serie EL CARDENALITO presenta de un total de 284, de las cuales solo 104 están relacionadas con la enseñanza del léxico; mientras que en la ENCICLOPEDIA GIRASOL, del total general, 1877 actividades, $452(24,08)$ son para el área de lengua y 202 están relacionadas con la enseñanza del léxico. Es importante destacar que en la COLECCIóN BICENTENARIO las actividades sobre el léxico se distribuyen de la siguiente manera 25 en $2^{\text {do }}$ grado, 23 para $4^{\text {to }}, 20$ en $3^{\text {ero, }} 16$ en $1^{\text {ero }}, 13$ en $5^{\text {to }}$ y 7 en $6^{\text {to }}$. En la ENCICLOPEDIA GIRASOL, la relación es la siguiente: 104 en $1^{\mathrm{er}}$ grado, 28 en $2^{\text {do }}, 24$ en $3^{\text {ro }}, 21$ en $6^{\text {to }}, 15$ en $5^{\text {to }}$ y 10 en $4^{\text {to }}$. No se pueden determinar las causas precisas de porque el número de actividades no se mantiene y decrece paulatinamente. Sería de interés establecerlas (ver gráfico 2). 


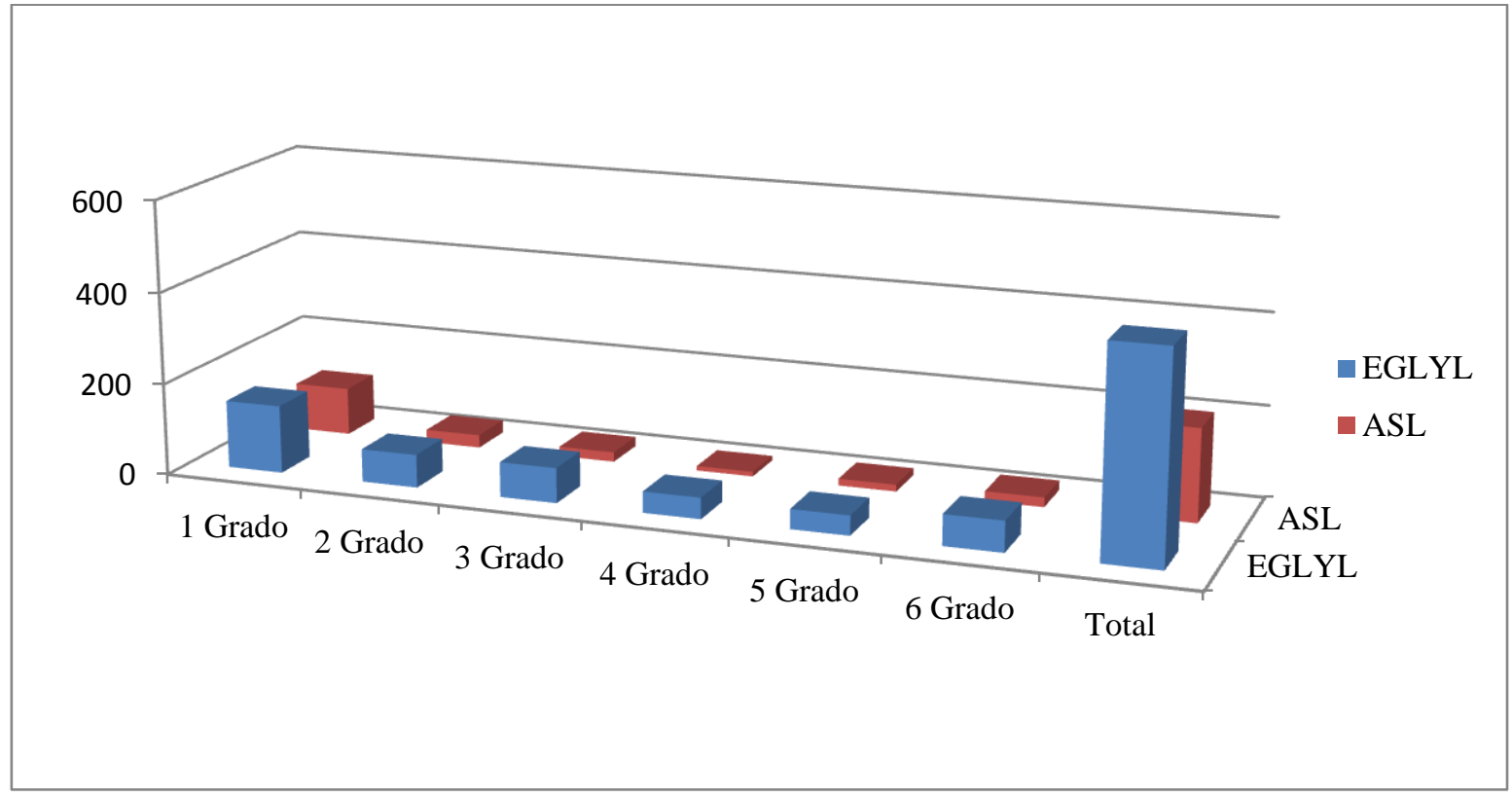

Gráfico 2. Total de actividades del área de Lengua y Literatura

Con la totalidad de las actividades sobre el léxico se procedió a determinar cuál era la proporción de actividades que permiten un aprendizaje explícito e implícito. Del total de 306 actividades, 179 (58,50\%) son explícitas y 127 (41,50\%) implícitas. Al discriminarlas por serie, en la ENCICLOPEDIA GIRASOL, la relación es la siguiente en $1^{\mathrm{er}}$ grado 30 $(28,85 \%)$ son explícitas y $74(71,15 \%)$ son implícitas; en $2^{\text {do }}$ grado $16(57,14 \%)$ frente a 12 (42,86\%); en $3^{\text {ero }} 13(54,16 \%)$ versus 11 $(45,84 \%)$; en $4^{\text {to }}$ se observa un equilibrio 5 son explícitas y 5 implícitas; en $5^{\text {to }} 6(40 \%)$ y
9 (60\%); y, finalmente, en $6^{\text {to }} 11(52,38 \%)$ procuran un aprendizaje explícitos y 10 (47,62\%), uno implícito. En El CARDENALITO, las relación es totalmente distinta, en $1^{\mathrm{er}}$ grado (16) y en $5^{\text {to }}$, todas las actividades son explícitas: 16 y 13, respectivamente. En 2 do, 24 (96,06\%); 3ero, 19 (95\%) y $6^{\text {to }} ; 6$ $(85,71 \%)$ son explícitas y solo hay 1 actividad implícita en cada grado. En lo que respecta a $4^{\text {to }}$ grado la relación de actividades explícita-implícitas es de $20(86,96 \%)$ y $3(13,04 \%)$ (Ver el gráfico 3 ). 


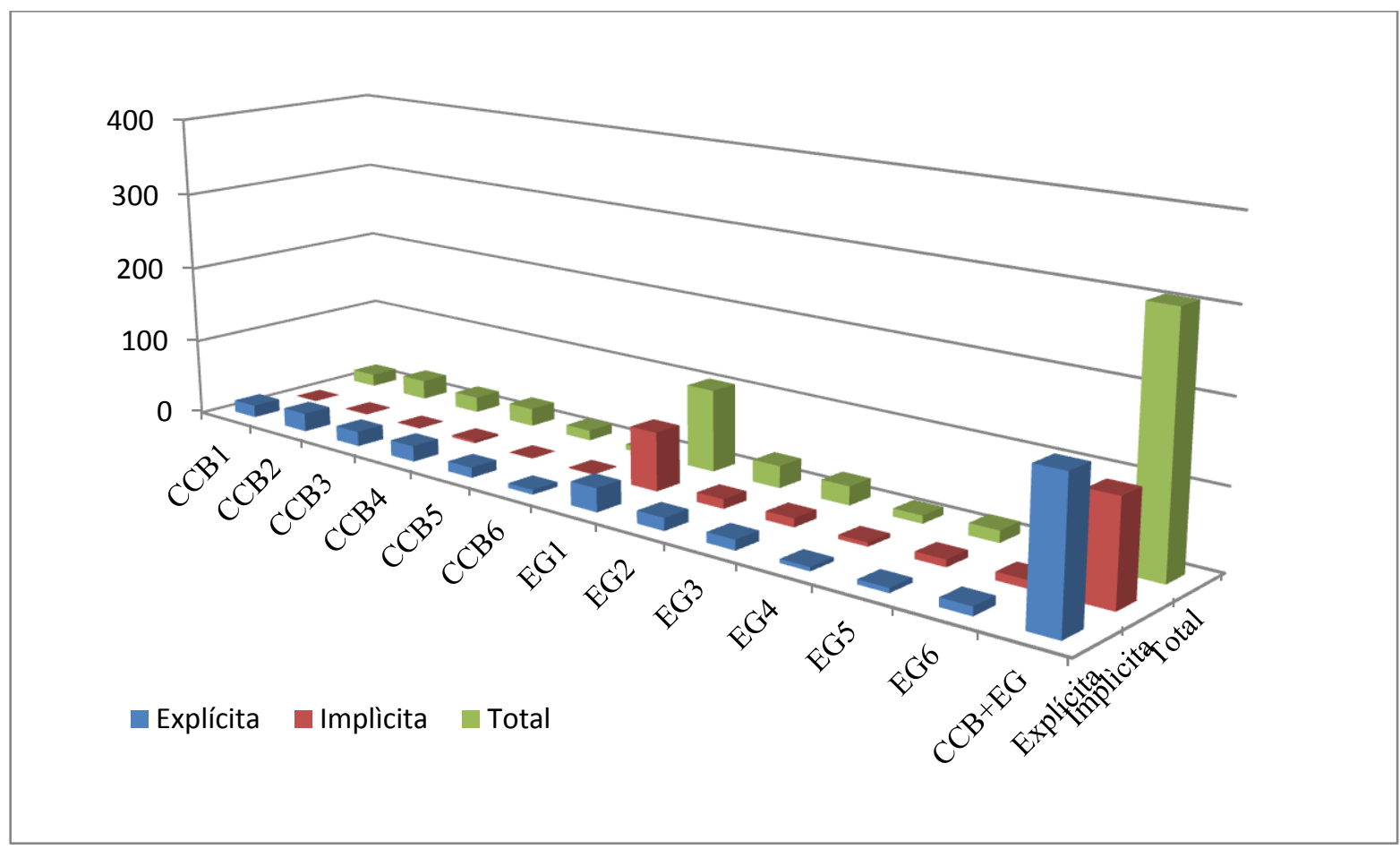

Gráfico 3. Tipos de actividades sobre el léxico

Estos resultados permiten afirmar que en la ENCICLOPEDIA GIRASOL se estudian como piezas aisladas pero también lo inserta en contexto para su mayor comprensión, tal como lo plantea Rodríguez (2006) mientras que en la serie El CARDEnAlito, el léxico se enseña como listas y glosarios aislados.

Al clasificar las actividades para el aprendizaje del léxico, a partir de la propuesta realizada por Moreno (2004), los resultados fueron los siguientes del total general de actividades $(308)^{2}$, 235 actividades son de convencionales, 40 son lúdicas y 33 son de constelación. Al discriminarlas por serie, en la ENCICLOPEDIA GIRASOL, 146 (71,93\%) son convencionales, 31 $(15,27 \%)$ son de constelación y $26(12,80 \%)$ son lúdicas; mientras que en El CARDENALITo, 89 son convencionales; 14 , lúdicas y 2 de constelación. Ninguna de las dos series recurren a las estrategias de dramatización (ver gráfico 4).

2 En el corpus de 306 actividades, dos actividades que podían ser ubicadas en dos tipos por lo que se maneja un total de 308 . 


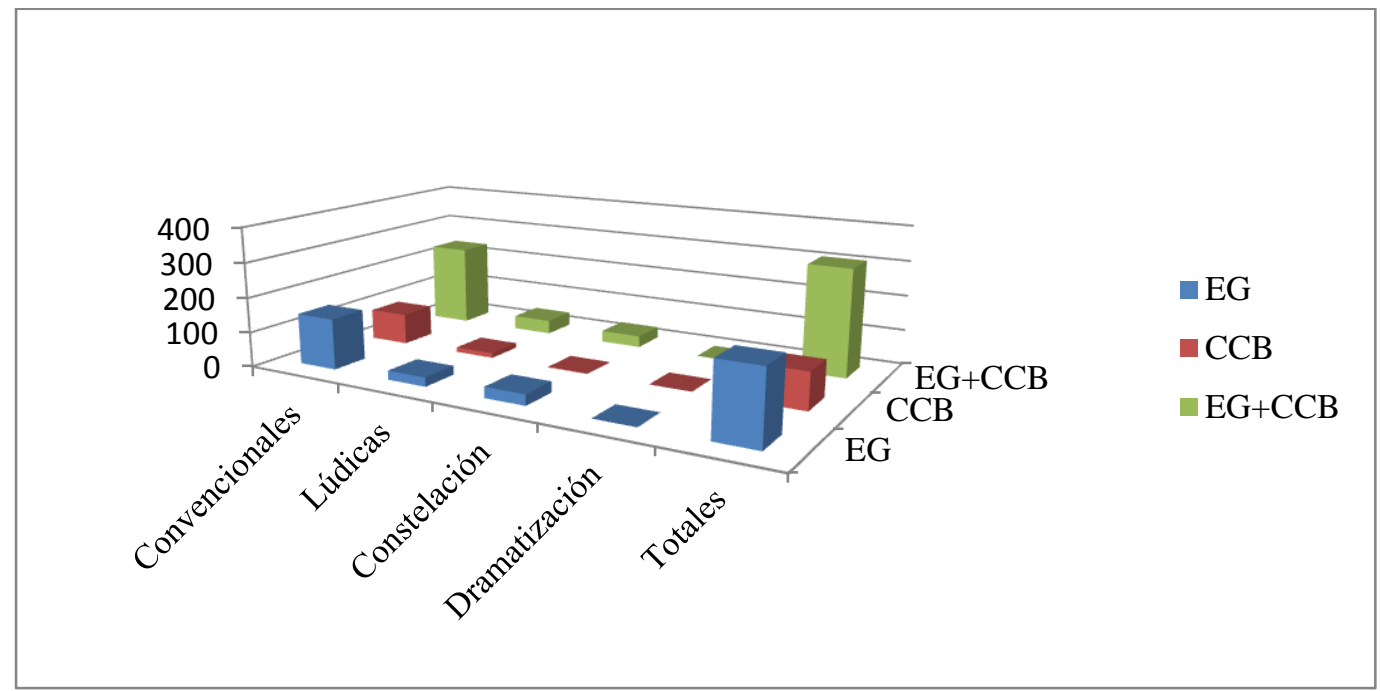

Gráfico 4. Tipos de actividades para la enseñanza del léxico

Los resultados permiten confirmar que ambas series se asocian con del Enfoque Tradicional para el aprendizaje del léxico ya que como plantea Moreno (2004) los ejercicios suelen ser "de resolución individual, poco contextualizados y algo mecánicos". En el caso de la ENCICLOPEDIA GIRASOL hay un intento por proponer actividades que consideren que el vocabulario no son piezas léxicas aisladas, sino redes o constelaciones de relaciones lingüísticas.

Una vez analizadas los tipos de actividades en general, se procedió a determinar cuáles eran los subtipos de actividades preferidas en cada tipo de la propuesta de Moreno (2004). En lo que respecta a las actividades convencionales, en las dos series:

1. 88 buscan la clasificación de las clases de palabras.

2. 38 su propósito es la construcción de palabras, bien sea mediante la unión de afijos y lexemas o mediante la unión de letras o sílabas.

3. 34 tiene como objetivo la construcción o reconstrucción de oraciones.

4. 34 solicitan que a una palabra se le dé una definición, o viceversa.
5. 18 piden que se le asigne a un dibujo una palabra.

6. 13 son ejercicios de relaciones semánticas.

7. 10 piden buscar palabras en el diccionario.

8. 8 requieren formar familias de palabras.

9. 1 hacer orden alfabético de palabras y determinar campos semánticos ${ }^{3}$, respectivamente.

Al comparar las actividades con los contenidos que establece el Currículo para el Subsistema de Educación Primaria, en lo que refiere al léxico, solo el 30\% de las actividades coinciden. Estos resultados son indicativos de que los manuales escolares manejan un conjunto de contenidos curriculares distinto a lo establecido por el Ministerio de Educación para cada grado del subsistema. Es interesante destacar que la fecha de publicación de los manuales de ambas serie es posterior al 2007, cuando se aprobó el referido documento. Además, la serie EL CARDENALITO es producida y distribuida gratuitamente por el Estado venezolano.

\footnotetext{
${ }^{3}$ la actividad no se enuncia con claridad su propósito.
} 
En ambas serie prevalecen los ejercicios relacionados con la clasificación de clases de palabras o partes de la oración, $50(56,18 \%)$ en la COLECCIÓn BICENTENARIO y 38 (26,03\%) en la ENCICLOPEDIA GIRASOL. Estos datos permiten confirmar lo planteado sobre el enfoque usado en ambos manuales escolares para el aprendizaje del léxico en la escuela primaria, así como afirmar que la ENCICLOPEDIA GIRASOL tiende a presentar más variedad de actividades que la serie EL CARDEnalito (ver gráfico 5).

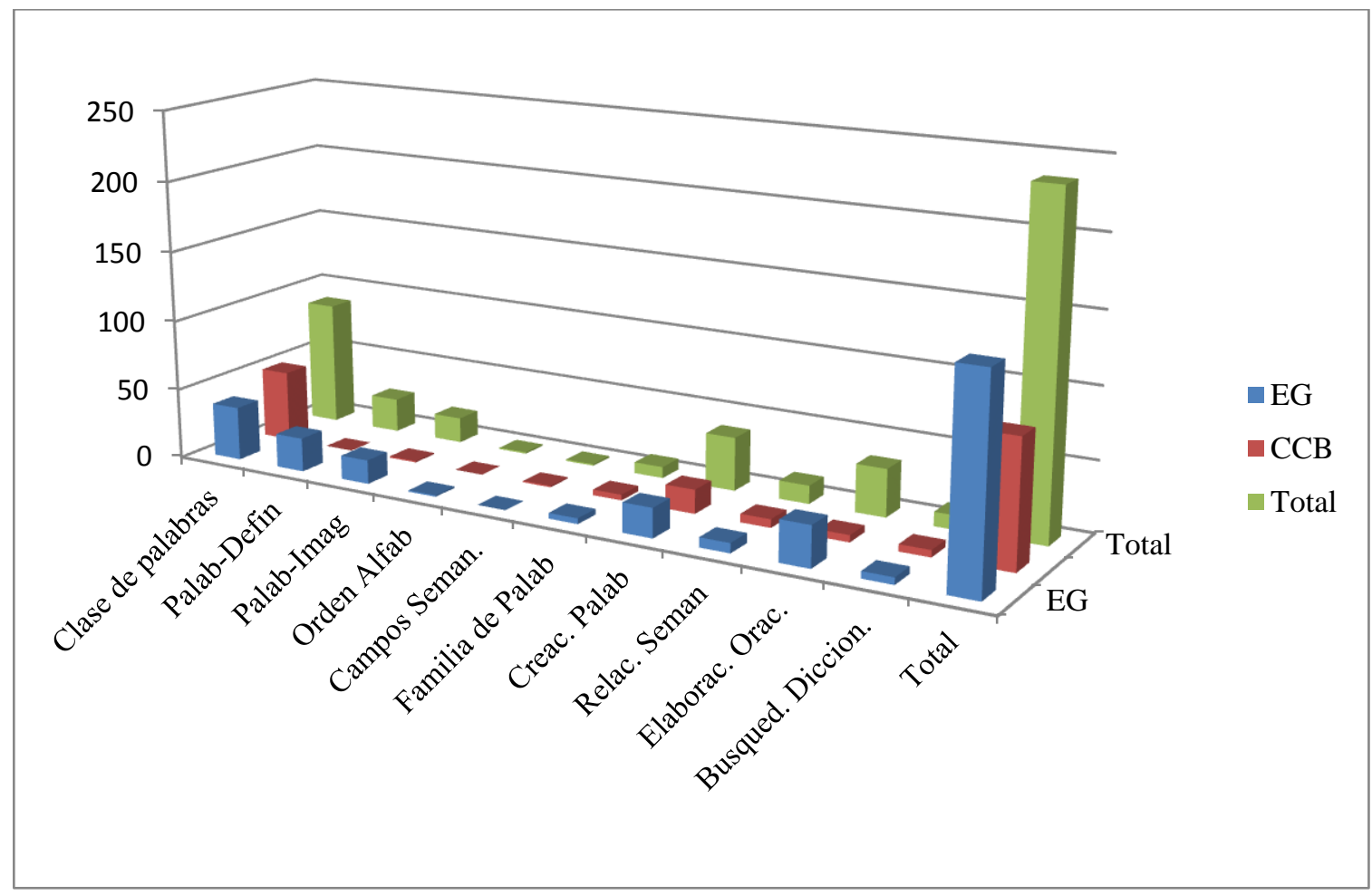

Gráfico 5. Tipo de actividades convencionales

En lo que respecta a las actividades lúdicas, del total de 40 actividades, 20 son de tipo sopa de letras, 9, palabras ocultas; 5 , damero; 2, dibujos o pictogramas y solo una actividad es de tipo crucigrama, cruzaletras, juego de memorias y pareamiento, respectivamente. Al discriminarla por serie, en la ENCICLOPEDIA
GIRASOL se prefieren los sopas de letras con 19 $(73,08 \%)$ actividades $y$ en la ColECCIón BICENTENARIO, la palabra oculta, $6(42,86 \%)$ y los dameros, 5 (35,72\%). Estos datos permiten confirmar lo concluido anteriormente sobre el aprendizaje del léxico en la escuela primaria (ver gráfico 6). 


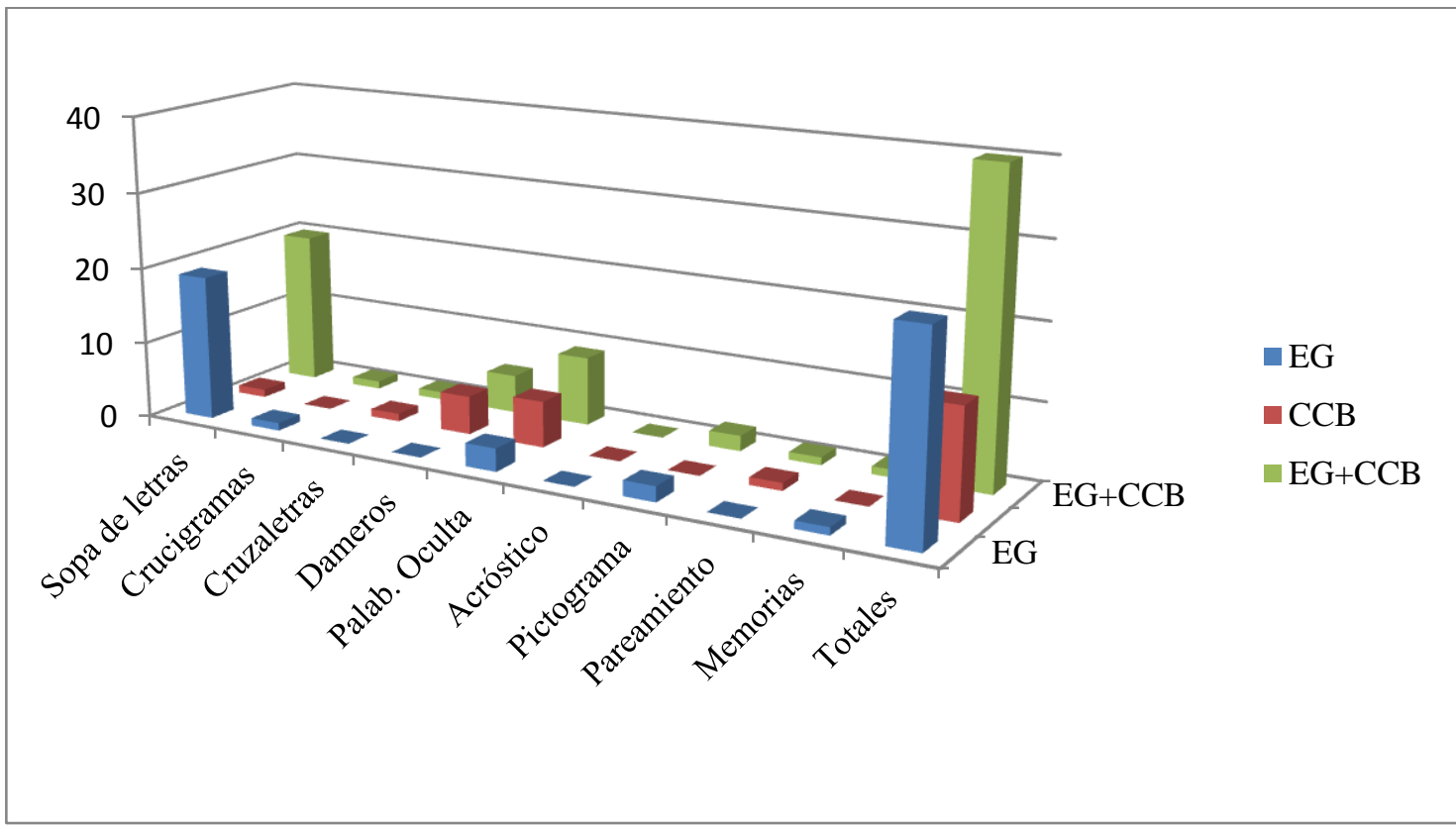

Gráfico 6. Tipos de actividades lúdicas

En cuanto a las actividades de constelación, de 33 actividades 30 son de tipo producción de textos y 3 de comprensión de escritos a partir de vocablos generadores. En el caso de la ENCICLOPEDIA GIRASOL se prefieren las actividades de producción, 28 (90,32\%), mientras que en EL CARDEnalito las de comprensión textual 3 (100\%). Indudablemente, estas actividades se acercan tímidamente a las verdaderas actividades de constelación, ya que tienen ciertos elementos que permitieron catalogarlas como tal. Todo esto ratifica el carácter tradicional que tiene el aprendizaje del léxico en la escuela primaria venezolana.

\section{CONCLUSIONES}

El desarrollo de la competencia léxica le permite al usuario de la lengua asir el conjunto de significados que engloba cada uno de las piezas léxicas. En el caso del léxico disciplinar, comprender y usar en forma precisa los sentidos que poseen los términos de cada disciplina. Desarrollar esta competencia en forma contextualizada contribuye al que el usuario pueda comprender los textos propios de un área académica o disciplina científica, además de poseer riqueza léxica que le permita producir escritos usando cada término con precisión y la neutralidad que los caracteriza. Por lo tanto, el aula debe promover estrategias y actividades contextualizadas que faciliten el dominio de ambas habilidades lingüísticas.

Los dos textos escolares analizados privilegian un aprendizaje del léxico por medio de actividades convencionales de resolución individual, poco contextualizadas, preferentemente relacionadas con la estructura morfológica de la palabra o su función sintáctica en frases $\mathrm{u}$ oraciones simples. Cuando se recurre a la semántica de los vocablos se hace aislada del texto. También se promueven actividades lúdicas, algunas individuales $y$ otras poco contextualizadas pero con cierto componente cooperativo. Estos resultados nos permiten inferir que el aprendizaje del léxico en la escuela primaria venezolana se asocia al enfoque tradicionalista de la enseñanza de la lengua, en donde el aprendizaje del léxico marginal y se hace mediante la memorización 
de listas y glosarios sin que se consideren las complejas redes de significados que se establecen en los textos o en el discurso. Todo esto obliga a un cambio significativo en las estrategias y actividades que use el maestro en el aula y a la producción de materiales didácticos que favorezcan el desarrollo de la competencia léxica, como subcomponente de la competencia comunicativa.

\section{REFERENCIAS}

Cassany, D; Luna, M y Sanz, G. (2001). Enseñar Lengua. Barcelona: Editorial Grao

Cortés, M. (2000). Guía para el profesor de idiomas. Didáctica del español y segundas lenguas. Barcelona: Ediciones Octaedro

Díaz, F y Hernández, G. (2002). Estrategias docentes para un aprendizaje significativo. Una interpretación constructivista. México: McGraw Hill

Giammatteo, M; Albano, H; Trombetta, A y Ghio, A. (2009). "El aprendizaje léxico: una propuesta de estrategias múltiples". En: M, Giammatteo, y H, Albano. (Coord), Lengua. Léxico, gramática y texto: Un enfoque para su enseñanza basado en estrategias múltiples (pp.17-43). Buenos Aires: Editorial Biblos

Gutiérrez, J y Rincón, L. (2008). Enciclopedia Girasol 1. Caracas: Editorial Girasol

Gutiérrez, J y Rincón, L. (2007). Enciclopedia Girasol 2. Caracas: Editorial Girasol

Gutiérrez, J y Rincón, L. (2013a). Enciclopedia Girasol 3. Caracas: Editorial Girasol

Gutiérrez, J y Rincón, L. (2013b). Enciclopedia Girasol 4. Caracas: Editorial Girasol

Gutiérrez, J y Rincón, L. (2014a). Enciclopedia Girasol 5. Caracas: Editorial Girasol

Gutiérrez, J y Rincón, L. (2014b). Enciclopedia Girasol 6. Caracas: Editorial Girasol

Ministerio del Poder Popular para la Educación. (2007a). Currículo del Subsistema de Educación Primaria. Caracas: Fundación Centro Nacional para el Mejoramiento de la Enseñanza de la Ciencia - CENAMEC

Ministerio del Poder Popular para la Educación. (2007b). Currículo Nacional Bolivariano. Diseño Curricular del Sistema Educativo Bolivariano. Caracas: Fundación Centro Nacional para el Mejoramiento de la Enseñanza de la Ciencia- CENAMEC

Moreno, J. (2004). Enseñar lengua desde un enfoque léxico. Glosas Didácticas. Revista Electrónica Digital. № 11. P: 162-168. Recuperado en: http://www.um.es/glosasdidacticas/doces/13moreno.pdf.

Muñoz, M. (2013a). El Cardenalito, Lengua y Literatura. Primer Grado. Caracas: Gobierno Bolivariano de Venezuela

Muñoz, M. (2014). El Cardenalito, Lengua y Literatura. Segundo Grado. Caracas: Gobierno Bolivariano de Venezuela

Muñoz, M. (2012a). El Cardenalito, Lengua y Literatura. Tercer Grado. Caracas: Gobierno Bolivariano de Venezuela

Muñoz, M. (2012b). Enciclopedia Girasol 1. Caracas: Editorial Santillana

Muñoz, M. (2013b). El Cardenalito, Lengua y Literatura. Cuarto Grado. Caracas: Gobierno Bolivariano de Venezuela

Muñoz, M. (2013c). El Cardenalito, Lengua y Literatura. Quinto Grado. Caracas: Gobierno Bolivariano de Venezuela

Muñoz, M. (2012b). El Cardenalito, Lengua y Literatura. Sexto Grado. Caracas: Gobierno Bolivariano de Venezuela

Nation, P. (2001). Learning Vocabualary in another Language, Cambridge University Press

Peñalver, M. (1991) La Lingüística y la enseñanza de la lengua española en el Bachillerato. Granada: Comares

Vidiella, M. (2012). El enfoque léxico en los manuales de ELE. Barcelona: Universitat de Barcelona: Memoria de Máster 